\title{
Ras-Related Protein Ral-B
}

National Cancer Institute

\section{Source}

National Cancer Institute. Ras-Related Protein Ral-B. NCI Thesaurus. Code C18293.

Ras-related protein Ral-B (206 aa, $\sim 23 \mathrm{kDa}$ ) is encoded by the human RALB gene. This protein plays a role in the regulation of GTP-dependent exocytosis. 